\title{
Investigating the Feasibility of a CMOS Camera in Developing a Shortwave Near Infrared Spectroscopy
}

\author{
Xien Yin Yap ${ }^{1}$, Kim Seng Chia $^{2^{*}}$, Hasan Ali Gamal Al-kaf ${ }^{3}$ \\ ${ }^{1}$ Faculty of Electrical and Electronic Engineering, \\ Universiti Tun Hussein Onn Malaysia, Parit Raja, Batu Pahat, MALAYSIA \\ ${ }^{2}$ Pusat Penyelidikan Shamsuddin Mikroelektronik Dan Nanoteknologi (Mint-Src), \\ Institut Kejuruteraan Integrasi, Universiti Tun Hussein Onn, Parit Raja, Johor, MALAYSIA \\ ${ }^{3}$ Department of Computer Science, \\ University of Science and Technology, Sana'a, YEMEN \\ *Corresponding Author
}

DOI: https://doi.org/10.30880/ijie.2020.12.08.020

Received 25 November 2019; Accepted 19 August 2020; Available online 31 August 2020

\begin{abstract}
Near infrared (NIR) sensing technology has been widely implemented in various areas as an alternative to substitute conventional wet chemistry analysis and sensing applications due to its non-invasive, green, and rapid measurement features. Recent researches indicate that a combination of NIR light emitted diode (LEDs) and photodiodes is promising to reduce the financial barriers to carry out NIR research in various applications. However, there is a challenge to detect and remove unwanted signals particularly ambient light and the changes of surrounding. This is because NIR signals are susceptible to the change of temperatures, moisture, and types of samples. This is worth to highlight that camera technology is feasible to remove unwanted backgrounds and insusceptible to the background for various applications e.g. face recognition, unmanned vehicle systems, and object classification. Therefore, this study aims to investigate the feasibility of a complementary metal-oxidesemiconductor (CMOS) camera in developing a shortwave NIR spectroscopy. Firstly, a slit, a NIR grating, and a CMOS camera were positioned and shielded in a black aluminum chassis. A total of six different parameters of the camera were investigated in this study, i.e. the exposure level, gain, white balance, brightness, sharpness, and saturation. Findings show that the CMOS camera with the optimal values of the exposure level and the gain could produce a good quality of NIR spectrum compared with default settling, in which, the signals were saturated. Thus, CMOS camera is feasible to be used to develop a low-cost NIR spectroscopy.
\end{abstract}

Keywords: Near infrared spectroscopy, CMOS camera, sensing technology, NIR LED

\begin{tabular}{ll}
\multicolumn{2}{l}{ Nomenclature } \\
NIR & Near infrared \\
LED & Light Emitted Diode \\
CMOS & Complementary Metal-Oxide-Semiconductor \\
USB & Universal Serial Bus
\end{tabular}

\section{Introduction}

Near infrared spectroscopy is a non-invasive and optical technology that uses the relative absorption of near infrared light (i.e. 780 to $2500 \mathrm{~nm}$ ). Near infrared spectroscopy has been successfully applied in the evaluation of food quality [1], [2]. Near infrared (NIR) sensing technology has been widely implemented in various areas e.g. medical [3][7], agriculture [2], [8]-[14], and industries [15]-[17] replace conventional wet chemistry analysis due to its non- 
invasive, green and rapid measurement features [18]-[20]. Multivariate calibration method such as partial least squares (PLS) has been used to establish the relationship between the near infrared spectra and the concentration [21]-[23]. Near infrared spectroscopy is a very sensitive device to the measurement environmental. The development of a better predictive model is always one of the key focus in NIR research to overcome unwanted effects from surrounding e.g. temperature and humidity that may affect the physicochemical composition of the samples [24].

Recently, new imaging system (e.g. hyperspectral imaging system) and reduced instrument size (e.g. handheld and portable instrument) are two key development areas in near infrared (NIR) sensing technology that enable real-time online monitoring and process control with relatively lower cost [24]. The latter may due to the development of light emitting diode (LED) technology and machine learning that has enable the possibility to design a low cost portable NIR sensor for various researches [25] e.g. predicting the internal quality of pineapples [26], predicting the ripeness of white grapes [27], tumor discrimination [28], and predicting the glucose level [29], [30]. The former, on the other hand, has enhanced the scope of analysis from one spot to the whole area of a sample. Consequently, the distribution of the component of interest across a sample e.g. the vitamin $\mathrm{C}$ distribution in fruit can be analyzed [31]. However, the financial barrier of conducting a NIR hyperspectral imaging research is significantly higher than that of conducting a NIR spectroscopic research.

In terms of electronic engineering perspective, there are two focuses in NIR research, i.e. the sensor development that involves electronic component design and signal preprocessing, and the predictive model or signal processing algorithms. This study focuses on the former, i.e. the sensor development and signal preprocessing. Most of the proposed low cost portable NIR sensors are using a combination of NIR LEDs and photodiodes, with or without a grating to disperse the spectrum. Despite its simplicity of the design structure, the feasibility of the design is highly affected by the surround effects, particularly the stray light and temperature. In fact, there is a challenge to detect and remove unwanted signals during NIR spectral acquisition because we have little idea about whether the light sensed by a photodiode is the desired signal or not. On the other hand, other sensing technology e.g. a complementary metaloxide-semiconductor (CMOS) camera that could provide more information about the signal is worthy to be investigated to measure NIR spectrum so that a better quality of NIR signal could be acquired. This is worth to highlight that camera technology is feasible to remove unwanted backgrounds and insusceptible to the background for various applications e.g. face recognition, unmanned vehicle systems, and object classification. Therefore, this study aims to investigate the feasibility of a CMOS camera in developing a shortwave near infrared spectroscopy.

\section{Experiment setup}

\subsection{Design framework}

Fig. 1 illustrates the framework of the proposed experiment to study the feasibility of a CMOS camera in developing a NIR sensor. The setup of the experiment can be classified into two parts, i.e. hardware development and the parameters study of the CMOS camera. Hardware development was about the arrangement of the slit, the grating and the camera as that further stated in Section 2.2. The parameters study of the camera was conducted using Matlab software that interfaced with the CMOS camera. A total of six parameters of the camera were studied i.e. the exposure, gain, white balance, saturation, brightness, and sharpness of the camera. The sequence to the parameter investigation of the camera to get the best spectrum without any over saturation as depicted in Fig. 2. At the end of the investigation, the best acquired spectrum with the best parameter was compared that acquired using the default setting. The performance analysis is presented in Section 2.3.

\subsection{Prototype Hardware Development}

Fig. 1 illustrates the experiment setup of the shortwave NIR spectroscopy that consists of a slit that allows a light beam passes through it and block unwanted light from unwanted directions; a NIR grating $(25 \times 25 \mathrm{~cm}$, Thorlab, Japan) that disperses the NIR light beam on the sensing element, and a CMOS camera that was used as the sensing element (i.e. JDEPC-OV04 VER:1.00 camera module, 1/4" CMOS sensor, OV2643, lens socket size of 8x8mm) to sense the intensity of the dispersed NIR spectrum. Consequently, each pixel of the camera measured the intensity of each wavelength from the dispersed NIR spectrum. A black aluminum L bar was used to build the structure to hold the slits, NIR grating, and the CMOS camera. The distance between the slit and grating was $14 \mathrm{~mm}$, while the distance between the grating and the camera was $13 \mathrm{~mm}$. These distances were tuned in ways that a signal with high resolution from the NIR light source was obtained. All components were shielded using a black aluminum chassis after the position of all components were fixed to reduce unwanted signals e.g. stray light from surrounding. 


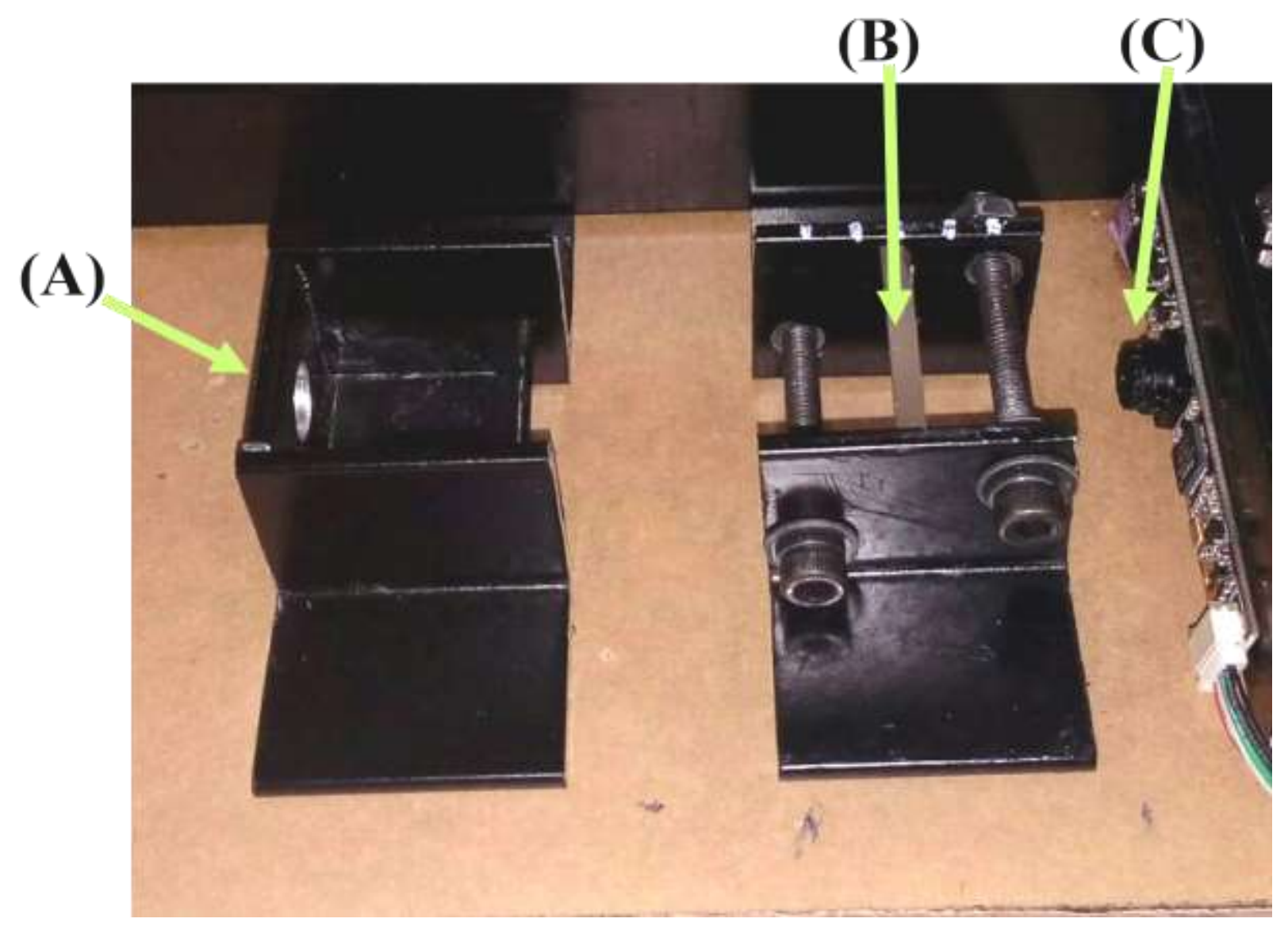

Fig. 1 - The experiment setup of the proposed shortwave NIR spectroscopy to acquire NIR spectrum: (a) a slit that blocks the light beams from unwanted directions, (b) a grating that disperses the light beam (from A to $B$ ) on the sensing element, and (c) a CMOS camera that was used as the sensing element

\subsection{Performance Analysis}

Next, the CMOS camera was connected to a laptop computer using a USB cable. Matlab software was used to interface the CMOS camera in order to modify the parameters of the camera and acquire the NIR spectrum. A total of six different parameters were investigated in this study, i.e. the exposure level, the gain, the white balance, the brightness, the sharpness, and the saturation of the camera. First, the parameters were set to manual setting instead of default auto setting. After that, a high precision NIR LED of $870 \mathrm{~nm}$ (Thorlab, Japan) was switch on and positioned in front of the designed NIR spectroscopy. Three different values of the exposure level (i.e. maximum, mean, and minimum) were used to evaluate their effects. The best exposure level was selected based on the quality of the image while other parameters were in default values. After that, the selection of the best value was proceeded from the gain, followed by the saturation, the white balance, the brightness, and then ended by the sharpness of the camera. Lastly, the proposed NIR spectroscopy was evaluated using another three high precision NIR LEDs (Thorlab, Japan) with different wavelengths of 780, 851, and $910 \mathrm{~nm}$. The flow of this performance analysis is depicted in Fig 2.

\section{Results and Discussion}

Fig 3. suggests that the sharpness and saturation parameters have no effect to the acquired NIR spectrum when their values were varied to maximum, mean, and minimum. These two parameters may only have effect on visible spectrum in terms of the amount of color and the clarity of an image, respectively. In the following section, the effects of different CMOS camera parameters of exposure level (Section 3.1), white balance (Section 3.2), gain and brightness (Section 3.3) were discussed when minimum, mean, and maximum values were used. After that, a comparison between the NIR spectrum with different wavelengths that acquired using the optimal parameters and default parameters were presented in Section 3.4. Lastly, Section 3.5 presents the overall performance analysis on the six parameters with respect to the acquired NIR signals. 


\section{Start}

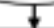

Arrange the distance between slit, grating and camera.

\begin{tabular}{|c|}
\hline $\begin{array}{c}\text { Capture the spectrum of the default } \\
\text { setting. }\end{array}$ \\
\hline Initial $\mathrm{i}=1$ \\
\hline $1=$ exposure level \\
2 = gain value \\
$3=$ white balance value \\
$4=$ saturation value \\
$5=$ brightness value \\
$6=$ Sharpness value \\
\hline
\end{tabular}
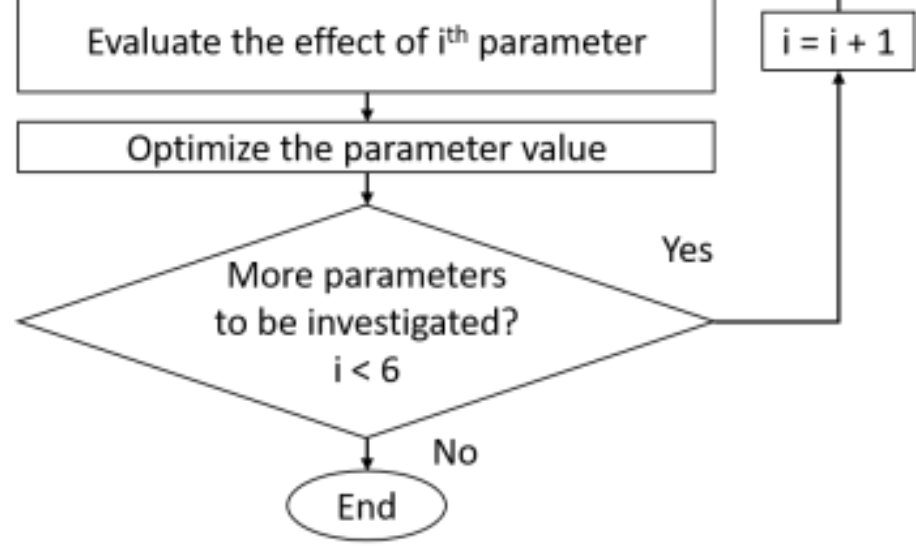

Fig. 2 - The flow of the effects of different exposure, gain, white balance, saturation, brightness, and sharpness values investigation

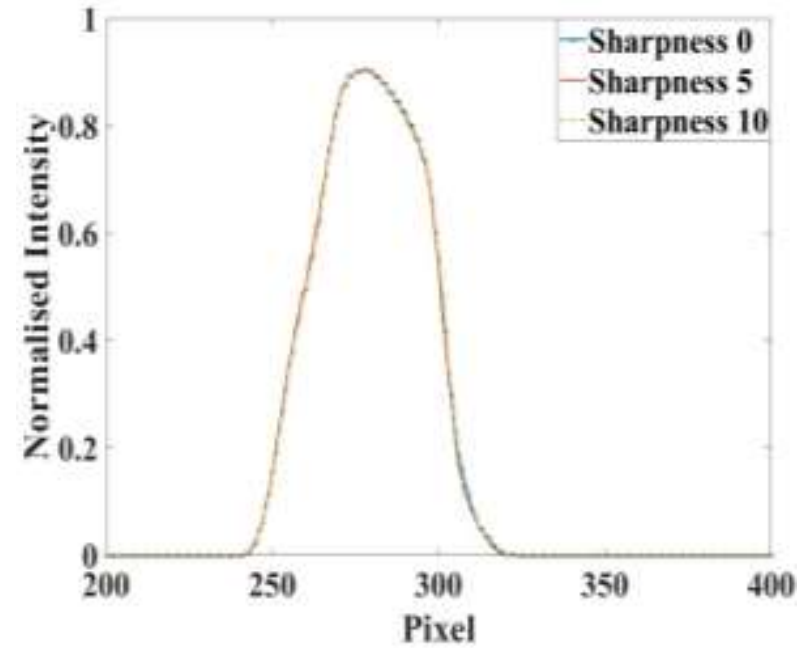

(a)

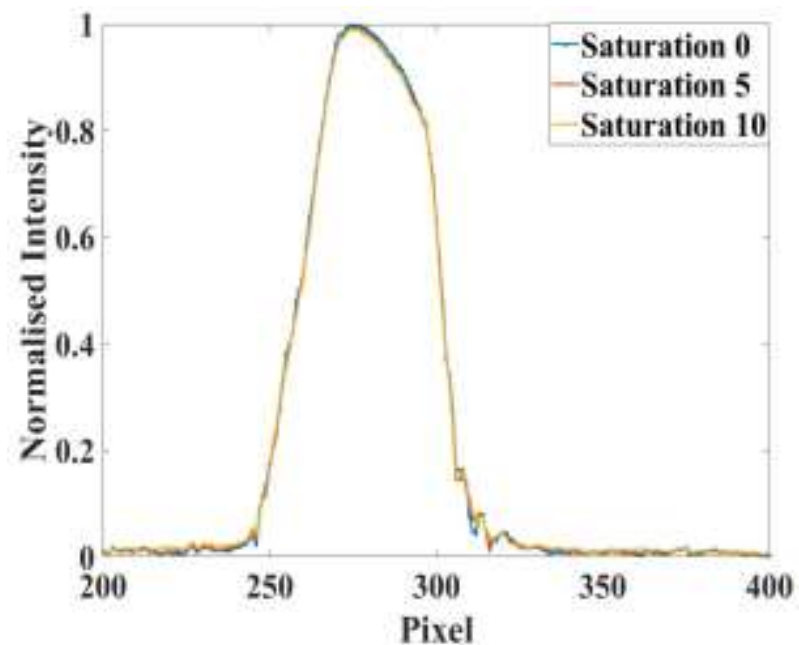

(b)

Fig. 3 - The effects of different CMOS camera parameters: (a) Sharpness and (b) saturation parameters. (Note. $\mathrm{Y}$-axis is the normalized intensity that is unitless, while $\mathrm{x}$-axis is the position of the pixel of the camera sensing element) 


\subsection{Exposure level}

Fig. 4 illustrates the spectrums with different exposure level. The exposure level value is in $\log$ base 2 seconds. By inspection, the exposure level of the CMOS camera should be set to the minimum value of -10 (or $2^{-10}$ seconds) in order to minimize the unwanted signals beyond the spectrum bandwidth. The gain of the CMOS camera will multiply the RGB colour values. Results suggest that the gain should be set at optimal value to avoid the signal to be under- and over-amplified. Since the acquired signal of the CMOS camera reached its maximum intensity with the minimum gain value, the minimum gain value (i.e. 32) was chosen as the best value to avoid over-amplifying the signals.

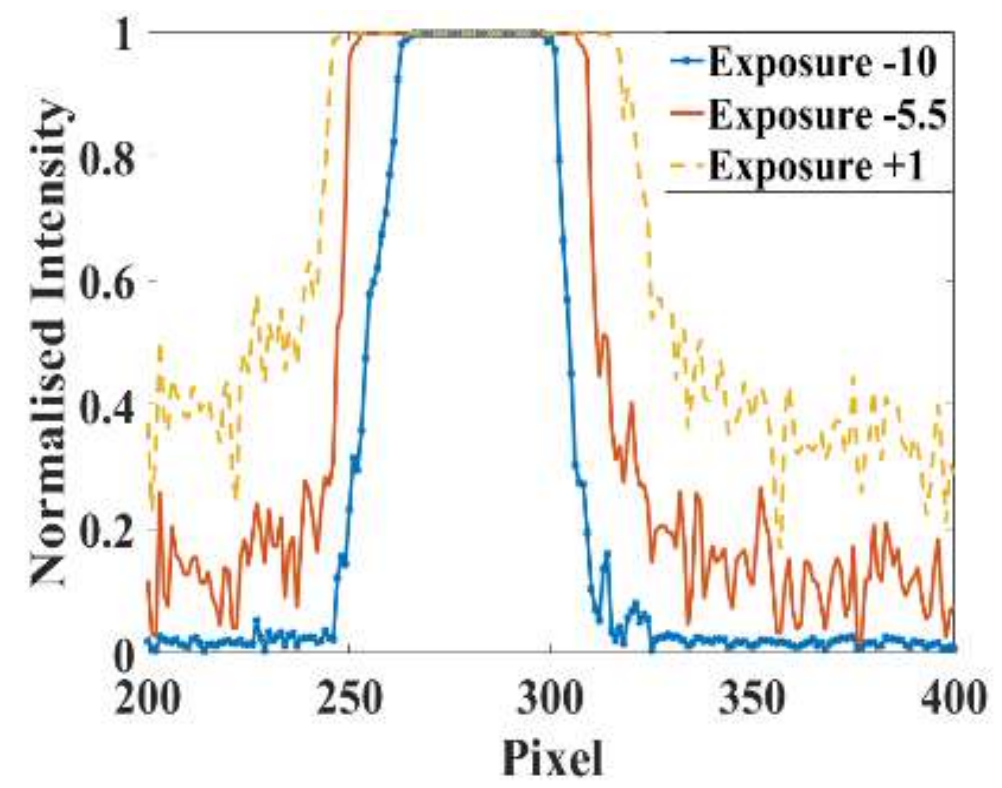

Fig. 4 - The effects of different exposure level. (Note. Y-axis is the normalized intensity that is unitless, while $\mathrm{x}$-axis is the position of the pixel of the camera sensing element)

\subsection{White balance}

Fig. 5 illustrates the spectrums with different white balance value. The white balance value appears have little effect on the maximum intensity of the NIR spectrum. Thus, the lowest white balance value of 2800 Kelvin (the color temperature) was chosen since it was able to remove some energy around the peak of the NIR spectrum that made the peak of the spectrum to be more obvious.

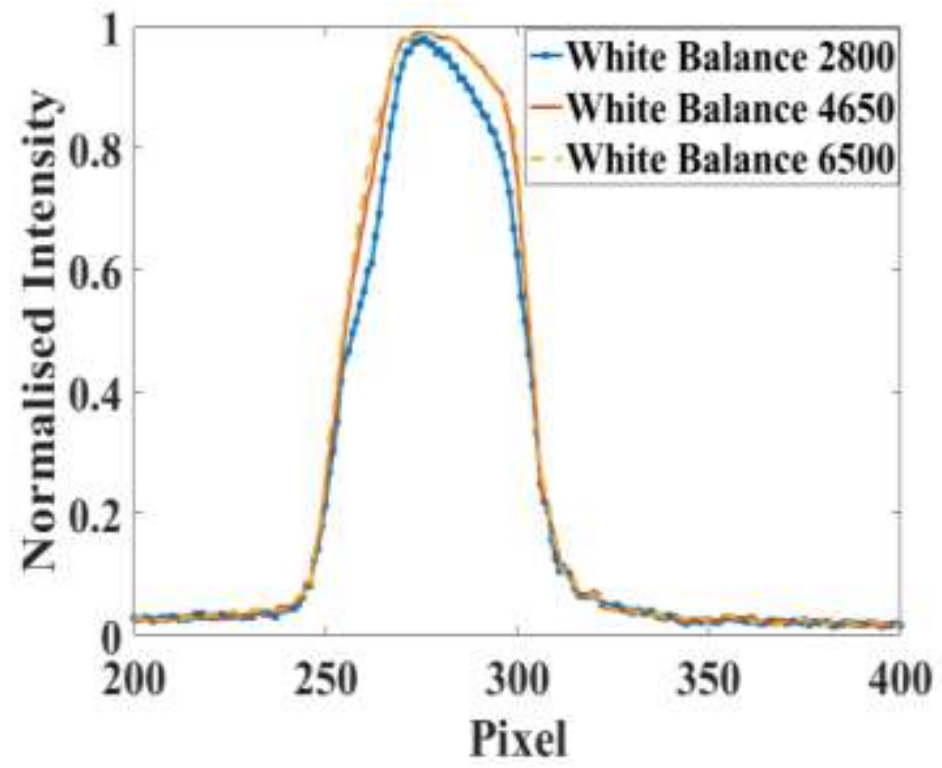

Fig. 5 - The effects of different white balance. (Note. Y-axis is the normalized intensity that is unitless, while $\mathrm{x}$-axis is the position of the pixel of the camera sensing element) 


\subsection{Brightness and Gain}

Fig. 6(a) illustrates the spectra with different brightness values, while Fig. 6(b) illustrates the spectrums with different gain values. The brightness level of the CMOS camera adjusts the amount of lighting on the sensing elements. Consequently, this parameter has similar effect as the gain parameter that affects the intensity of the acquired NIR spectrum. Therefore, both gain parameter and brightness level can be tuned together to adjust the intensity of the acquired signals. These two parameters are useful to ensure optimal signal was acquired to optimize the signal-to-noise ratio and to saturation in which useful information cannot be quantified correctly.

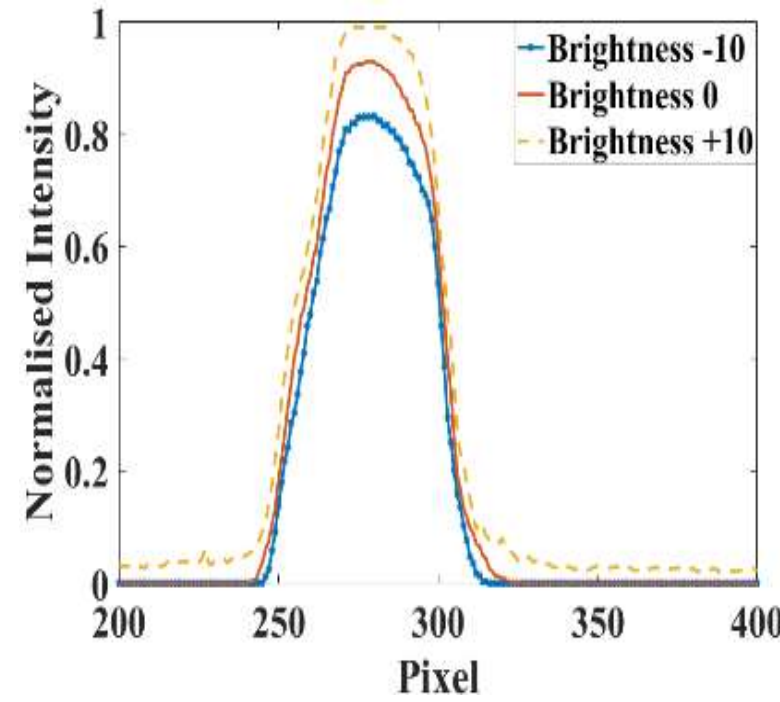

(a)

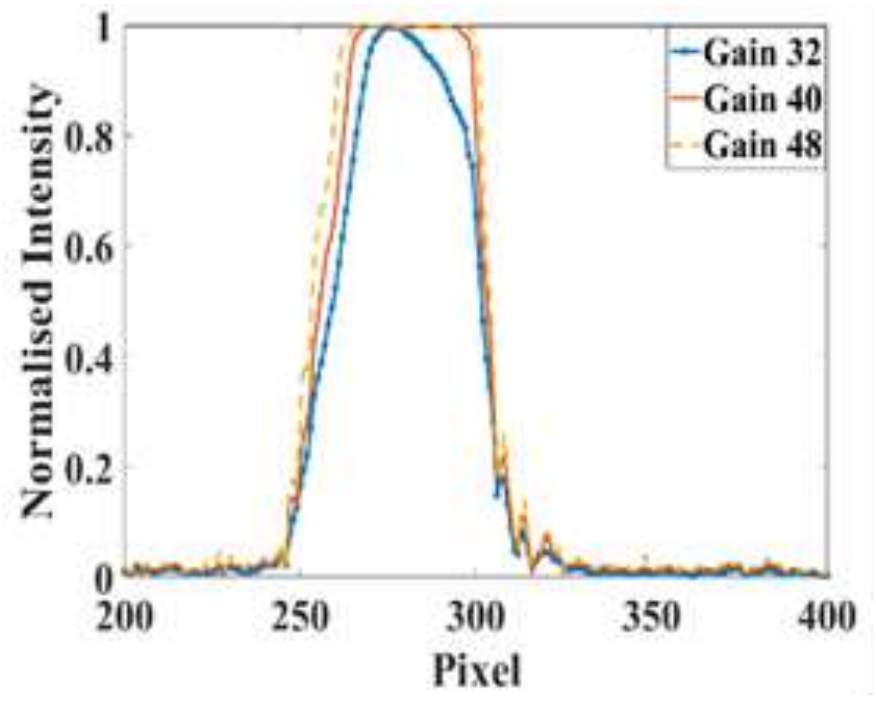

(b)

Fig. 6 - The effects of different (a) brightness and (b) gain. (Note. Y-axis is the normalized intensity that is unitless, while $x$-axis is the position of the pixel of the camera sensing element)

\subsection{Effects of Different Wavelengths}

Fig. 7 shows the acquired NIR spectra from the NIR LEDs with different wavelengths when the CMOS camera used the optimal parameters setting. A correct sequence of the NIR spectral peaks, i.e. from 780 to $910 \mathrm{~nm}$, suggests that the setup of proposed NIR spectroscopy is promising to be further developed to detect the peak value of the shortwave NIR spectrum that covers the range from 750 to $1000 \mathrm{~nm}$. Different intensities were sensed among different wavelengths. This is expected because the sensitivity (i.e. voltage per light intensity) of the sensing element is different along the spectrum, and the light intensity that emitted from the NIR LEDs were different.

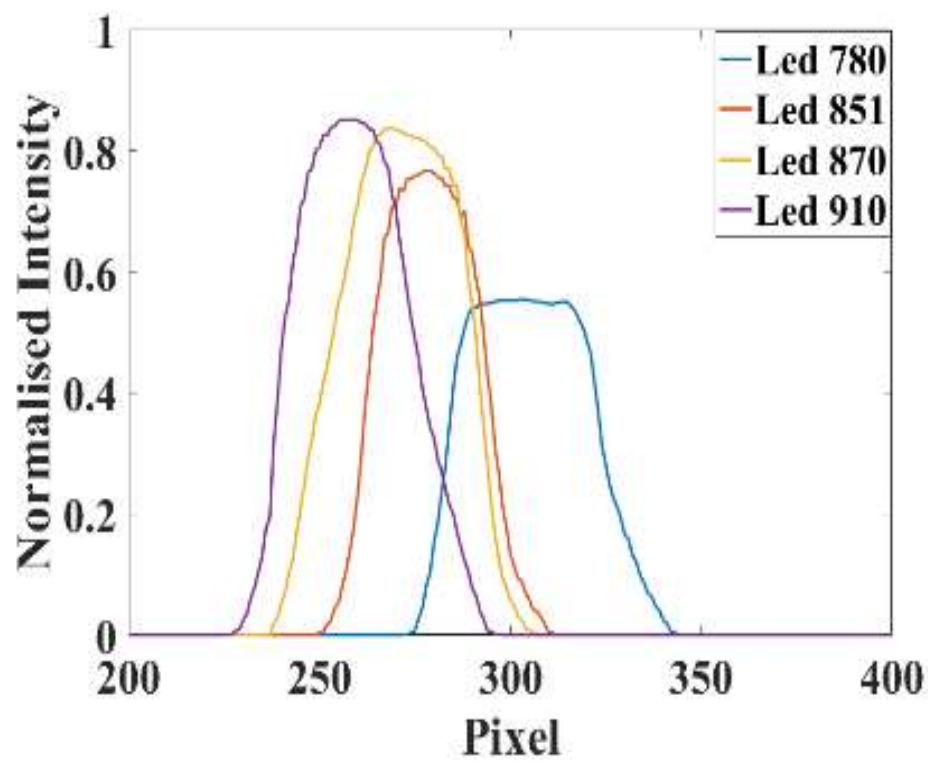

Fig. 7 - The shortwave near infrared spectrum that measured using the designed settling. (Note. Y-axis is the normalized intensity that is unitless, while $x$-axis is the position of the pixel of the camera sensing element) 


\subsection{Overall Performance Analysis}

Table 1 summarize the optimal values of each parameters that obtained from the above analysis, compared with the default value. The results indicate that both exposure level and white balance were changed automatically in the default settling. This could be due to the camera was designed to capture image in which exposure level and white balance were needed to be tuned to ensure the acquired image was clear during different environment conditions. However, this default settling is inappropriate to the proposed application i.e. acquired NIR spectrum because the change of the intensity must be affected by the components of interest instead of the environment changes

\begin{tabular}{ccc} 
Table 1 - The difference between default and optimal values of each parameter \\
\hline Parameter & Default value & Optimal value \\
\hline Exposure level & Auto & -10 \\
Gain & 48 & 32 \\
White balance & Auto & 2800 Kelvin \\
Brightness & 10 & 10 \\
Sharpness & 0 & No care \\
Saturation & 0 & No care \\
\hline
\end{tabular}

Fig. 8 depicts a comparison between the acquired spectrum that used the optimal parameters settling and that used the default parameters settling of the CMOS camera. Apparently, the CMOS camera that used its auto parameters settling was unable to sense the NIR spectrum due to unwanted signals that overlapped the desired signals. This may be due to the fact the auto settling was trying to get a good visible spectrum.

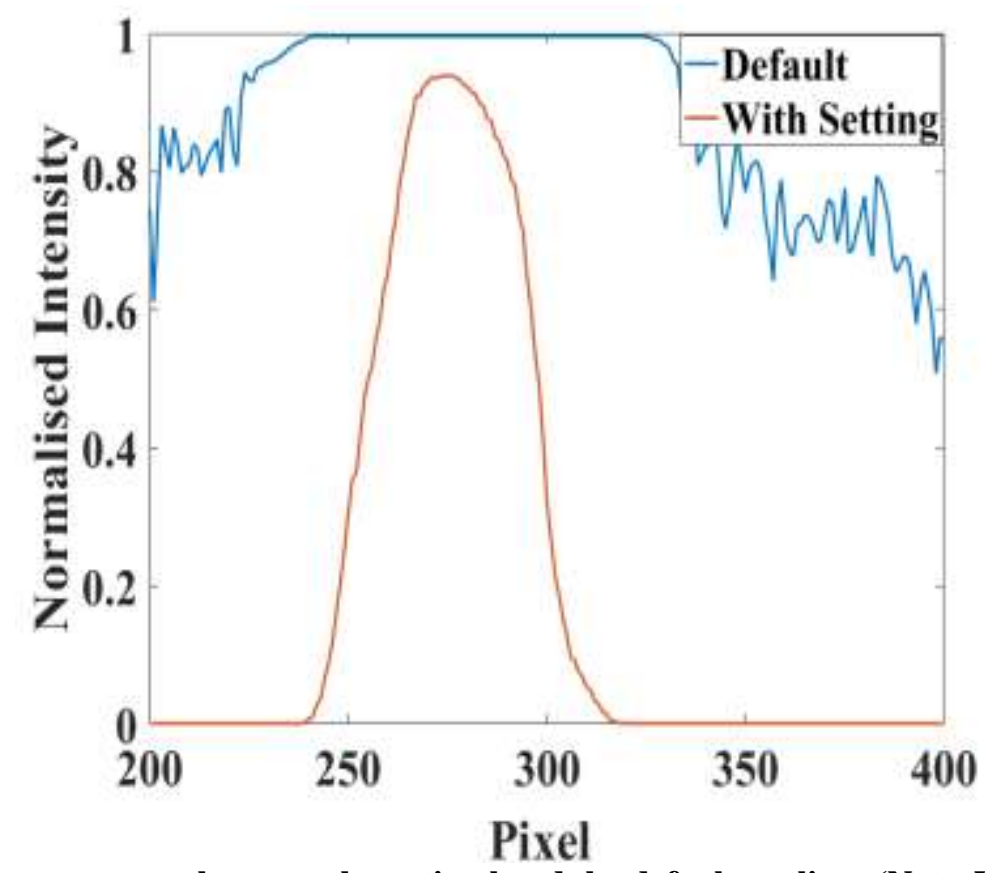

Fig. 8 - The comparison spectrum between the optimal and the default settlings (Note. Y-axis is the normalized intensity that is unitless, while $\mathrm{x}$-axis is the position of the pixel of the camera sensing element)

Table 2 summarizes the acquired normalized intensity when different parameters of the camera were discussed in the previous sections. The mean value of each parameter was used as a reference, thus we state "no applicable" at the observation column. On the other hand, the effects of different values (maximum and minimum) were observed and recorded. When tuning the exposure level, gain and white balance, the spectrum was over saturated but the bandwidth of the spectrum become smaller at $80 \%$ of the peak wavelength. The spectrum was not over saturated when the brightness value was decreased. The intensity of the spectrum was reduced from over-saturated to 0.83 when the brightness was varied from +10 to -10 . There is the combination to reduce the light intensity. This is because we need to tune all exposure, white balance, gain and brightness in order to provide a quality NIR signal that cannot be achieved by tuning a single parameter only. The sequence in tuning the parameters did not affect the results. The experiments indicate that the sharpness and saturation had no effect to the acquired NIR spectrum. In other words, the intensity of the acquired spectra was the same with that acquired using different setting values. 
Table 2 - The effects of the parameters values of the CMOS camera when it was used to acquired NIR spectrum that emitted from NIR LED

\begin{tabular}{ccccc} 
Parameter & $\begin{array}{c}\text { Setting } \\
\text { value }\end{array}$ & $\begin{array}{c}\text { Normalized } \\
\text { Intensity }\end{array}$ & $\begin{array}{c}\text { Peak Wavelength } \\
\text { (pixel) }\end{array}$ & Observation \\
\hline \multirow{2}{*}{ Exposure level } & -10 & Over Saturated & 280 & The acquired intensity is reduced. \\
& -5.5 & Over Saturated & 280 & Reference value. No applicable. \\
& +1 & Over Saturated & 280 & The acquired intensity is increased. \\
Gain & 32 & Over Saturated & 275 & The acquired intensity is reduced. \\
& 40 & Over Saturated & 282 & Reference value. No applicable. \\
White balance & 48 & Over Saturated & 282 & The acquired intensity is increased. \\
& 2800 & Over Saturated & 275 & The acquired intensity is reduced. \\
Brightness & 6550 & Over Saturated & 275 & Reference value. No applicable. \\
& -10 & Over Saturated & 275 & The acquired intensity is increased. \\
& 0 & 0.83 & 277 & The acquired intensity is reduced. \\
Sharpness & +10 & Over Saturated & 278 & Reference value. No applicable. \\
& 0 & 0.90 & 278 & The acquired intensity is increased. \\
& 5 & 0.90 & No effect is observed. \\
Saturation & 10 & 0.90 & 278 & Reference value. No applicable. \\
& 0 & Over Saturated & 276 & No effect is observed. \\
& 10 & Over Saturated & 276 & No effect is observed.
\end{tabular}

\section{Conclusion}

This study shows that the CMOS camera is a promising sensing element to be used to sense NIR spectrum and subsequent to develop a low-cost NIR spectroscopy. This is because various signal processing can be implemented to improve the quality of the acquired NIR spectrum and to remove unwanted signals from surrounding. Particularly, the exposure level and the gain of a CMOS should be properly tuned to produce a better quality of NIR spectrum by avoiding over saturation. Also, results suggest that the sharpness and saturation parameters of the CMOS camera have no effect to the acquired NIR spectrum. Findings show that the CMOS camera with the optimal values of the exposure level and the gain could produce a good quality of NIR spectrum, compared with that used default parameter values. However, a further investigation is needed to verify whether other parameters e.g. contrast, Gamma, Hue, and Iris have any positive effect to the acquired NIR spectrum, and the important signals that embedded in NIR spectrum during NIR research could be mainly retained by the proposed NIR spectroscopy.

\section{Acknowledgement}

The authors would like to acknowledge Faculty of Electrical and Electronic Engineering, Universiti Tun Hussein Onn Malaysia for providing facilities for this study. We acknowledge the funding from Geran Penyelidikan Pascasiswazah Fasa 2/2018 (GPPS) (H313) under Research Management Centre (RMC) in UTHM in completing the research.

\section{References}

[1] M. C. Alamar, E. Bobelyn, J. Lammertyn, B. M. Nicolaï, and E. Moltó. (2007). Calibration transfer between NIR diode array and FT-NIR spectrophotometers for measuring the soluble solids contents of apple. Postharvest Biol. Technol., 45, 38-45.

[2] C. J. Hayes, K. B. Walsh, and C. V. Greensill. (2016). Improving calibration transfer between shortwave near infrared silicon photodiode array instruments. J. Near Infrared Spectrosc., 24, 59-68.

[3] J. Peng, S. Peng, A. Jiang, and J. Tan. (2011). Near-infrared calibration transfer based on spectral regression. Spectrochim. Acta - Part A Mol. Biomol. Spectrosc., 78, 1315-1320. 
[4] M. Boiret, L. Meunier, and Y. M. Ginot. (2011). Tablet potency of Tianeptine in coated tablets by near infrared spectroscopy: Model optimisation, calibration transfer and confidence intervals. J. Pharm. Biomed. Anal., 54, $510-516$.

[5] V. H. da Silva, J. J. da Silva, and C. F. Pereira. (2017). Portable near-infrared instruments: Application for quality control of polymorphs in pharmaceutical raw materials and calibration transfer. J. Pharm. Biomed. Anal., 134, 287-294.

[6] W. R. Chen, J. Bin, H. M. Lu, Z. M. Zhang, and Y. Z. Liang. (2016). Calibration transfer via an extreme learning machine auto-encoder. Analyst, 141, 1973-1980.

[7] Y. Hu, S. Peng, Y. Bi, and L. Tang. (2012). Calibration transfer based on maximum margin criterion for qualitative analysis using Fourier transform infrared spectroscopy. Analyst, 137, 5913-5918.

[8] Y. Sulub, R. LoBrutto, R. Vivilecchia, and B. W. Wabuyele. (2008). Content uniformity determination of pharmaceutical tablets using five near-infrared reflectance spectrometers: A process analytical technology (PAT) approach using robust multivariate calibration transfer algorithms. Anal. Chim. Acta, 611, 143-150.

[9] L. Ni, M. Han, S. Luan, and L. Zhang. (2019). Screening wavelengths with consistent and stable signals to realize calibration model transfer of near infrared spectra. Spectrochim. Acta - Part A Mol. Biomol. Spectrosc., 206, 350-358.

[10] Y. Mou, L. Zhou, S. Yu, W. Z. Chen, X. Zhao, and X. You. (2016). Robust calibration model transfer. Chemom. Intell. Lab. Syst., 156, 62-71.

[11] D. V. Poerio and S. D. Brown. (2018). Dual-Domain Calibration Transfer Using Orthogonal Projection. Appl. Spectrosc., 72, 378-391.

[12] J. Zhang, C. Guo, X. Cui, W. Cai, and X. Shao. (2018). A two-level strategy for standardization of near infrared spectra by multi-level simultaneous component analysis. Anal. Chim. Acta.

[13] B. Yu, H. Ji, and Y. Kang. (2016). Standardization of near infrared spectra based on multi-task learning. Spectrosc. Lett., 49, 23-29.

[14] N. K. Wijewardane, Y. Ge, S. Wills, and T. Loecke. (2016). Prediction of Soil Carbon in the Conterminous United States: Visible and Near Infrared Reflectance Spectroscopy Analysis of the Rapid Carbon Assessment Project. Soil Sci. Soc. Am. J., 80, 973.

[15] F. Araújo, N. Cavalcante, J. M. Amigo, and M. Fernanda. (2017). Standardization from a benchtop to a handheld NIR spectrometer using mathematically mixed NIR spectra to determine fuel quality. Anal. Chim. Acta, 954, 32-42.

[16] J. B. Cooper, C. M. Larkin, and M. F. Abdelkader. (2011). Virtual standard slope and bias calibration transfer of partial least squares jet fuel property models to multiple near infrared spectroscopy instruments. J. Near Infrared Spectrosc., 19, 139-150.

[17] J. B. Cooper, C. M. Larkin, and M. F. Abdelkader. (2011). Calibration transfer of near-IR partial least squares property models of fuels using virtual standards. J. Chemom., 25, 496-505.

[18] M. N. E. M. Idrus, K. S. Chia, H. M. Sim, and H. A. G. Al-kaf. (2018). Artificial neural network and Savitzky Golay derivative in predicting blood hemoglobin using near-infrared spectrum. International Journal of Integrated Engineering, 10, 112-119.

[19] B. C. Deng et al. (2016). A bootstrapping soft shrinkage approach for variable selection in chemical modeling. Anal. Chim. Acta, 908, 63-74.

[20] M. Mancini et al. (2018). Near infrared spectroscopy for the discrimination between different residues of the wood processing industry in the pellet sector. Fuel, 217, 650-655.

[21] K. D. T. M. Milanez, A. C. Silva, J. E. M. Paz, E. P. Medeiros, and M. J. C. Pontes. (2016). Standardization of NIR data to identify adulteration in ethanol fuel. Microchem. J., 124, 121-126.

[22] B. Malli, A. Birlutiu, and T. Natschläger. (2017). Standard-free calibration transfer - An evaluation of different techniques. Chemom. Intell. Lab. Syst., 161, 49-60.

[23] Y. Liu, W. Cai, and X. Shao. (2014). Standardization of near infrared spectra measured on multi- instrument. Anal. Chim. Acta, 836, 18-23.

[24] S. Brown. (2015). Analysis of the State of the Art : Near-Infrared Spectroscopy. Spectroscopy, 30, 1-7.

[25] K. S. Chia and Y. P. Tan,. (2017). Design and development of a shortwave near infrared spectroscopy using NIR LEDs and regression model. Int. J. Electr. Comput. Eng., 7, 3070-3075.

[26] M. N. H. Jam and K. S. Chia. (2017). Investigating the relationship between the reflected near infrared light and the internal quality of pineapples using neural network. Int. J. Adv. Sci. Eng. Inf. Technol., 7, 1389-1394.

[27] V. Giovenzana, R. Civelli, R. Beghi, R. Oberti, and R. Guidetti. (2015). Testing of a simplified LED based vis/NIR system for rapid ripeness evaluation of white grape (Vitis vinifera L.) for Franciacorta wine. Talanta, 144, 584-591.

[28] A. Bogomolov et al.. (2017). Development and testing of an LED-based near-infrared sensor for human kidney tumor diagnostics. Sensors (Switzerland), 17, 1-17. 
[29] J. Yadav, A. Rani, V. Singh, and B. M. Murari. (2014). Near-infrared LED based non-invasive blood glucose sensor. 2014 Int. Conf. Signal Process. Integr. Networks, SPIN 2014, (pp. 591-594).

[30] K. S. Chia, N. A. S. Suarin, and S. F. Z. M. Fuzi. (2017). Prediction of glucose concentration using near infrared light and adaptive linear neuron. Proc. - 2017 IEEE 13th Int. Colloq. Signal Process. its Appl. CSPA pp. 35-38.

[31] C. Malegori, S. Grassi, E. J. N. Marques, S. T. de Freitas, and E. Casiraghi.(2016). Vitamin C distribution in acerola fruit by near infrared hyperspectral imaging. J. Spectr. Imaging, 5, 6-9. 